\title{
Metabolism of Wogonoside by Human Fecal Microflora and Its Anti-pruritic Effect
}

\author{
Hien-Trung TrinH ${ }^{1}$, Seo-Young JANG ${ }^{2}$, Myung Joo HAN ${ }^{2}$, Ho-Young KAWK ${ }^{3}$, Nam-In BAEK ${ }^{3}$, and Dong-Hyun KIM ${ }^{1, *}$ \\ ${ }^{1}$ Department of Pharmaceutical Science and Department of Life and Pharmaceutical Sciences, ${ }^{2}$ Department of Food and Nutrition, \\ ${ }^{3}$ Graduate School of Biotechnology and PMRC, Kyung Hee University, Seoul 130-701, Republic of Korea
}

(Received February 26, 2009; Revised April 6, 2009; Accepted April 8, 2009)

\begin{abstract}
To understand the relationship between the metabolism of wogonoside from the rhizome of Scutellaria baicalensis, and its anti-pruritic effect, we anaerobically incubated it with human fecal microflora, identified its metabolite identified, and investigated its anti-pruritic effect in compound 48/80 or histamineinduced pruritic mice. Wogonoside was metabolized to wogonin, with metabolic activity of $6.9 \pm 5.1 \mathrm{nmol} / \mathrm{h} /$ $\mathrm{mg}$ wet weight of fecal microflora. Orally administered wogonoside had more potent anti-scratching behavioral effect in compound $48 / 80$ or histamine-treated mice than intraperitoneally treated one, apart from orally administered its metabolite, wogonin, which was more potent than the orally administered one. Wogonoside showed more potent anti-pruritic effects when administered at $5 \mathrm{~h}$ prior to the pruritic agent treatment than when administered at $1 \mathrm{~h}$ before. However, wogonin orally administered $1 \mathrm{~h}$ before the treatment with pruritic agents showed a more potent anti-pruritic effect than when treated at $5 \mathrm{~h}$ before. Orally administered wogonoside may be metabolized to wogonin in the intestine and its anti-scratching behavioral effect may be dependent on its metabolism by intestinal microflora.
\end{abstract}

Keywords: Scutellaria baicalensis, wogonoside, wogonin, pruritus, metabolism, intestinal microflora

\section{INTRODUCTION}

Most herbal medicines are orally administered to humans. Their components are therefore inevitably brought into contact with intestinal microflora in the alimentary tract. Many components may be transformed by the intestinal bacteria before absorption from the gastrointestinal tract. Studies on the metabolism of the components by human intestinal microflora are of a great importance to an understanding of their biological effects (Kobashi and Akao, 1997; Akao et al., 1998).

The rhizome of Scutellaria baicalensis (SB), which contains baicalin and wogonoside as the main constituents, has long been used in China, Japan and Korea as a traditional medicine and functional food for inflammation, fever, hepatitis, allergic disease, hypertension, etc (Zhu, 1998; Wu et al., 2005). These constituents exhibit anti-inflammatory, antiallergic, anti-oxidant, and hepatoprotective, and antitumor effects (Chou et al., 2003; Jang et al., 2003;

*Corresponding author

Tel: +82-2-961-0374 Fax: +82-2-957-5030

E-mail: dhkim@khu.ac.kr
Lim, 2003; Kim et al., 2005). These components are poorly absorbed from the gastrointestinal tract in its native form due to their hydrophilic properties and must be hydrolyzed by intestinal microflora in the intestine to their aglycones in human and rats (Akao et al., 2000; Yim et al., 2004). The absorbed metabolite, baicalein, is subsequently conjugated to baicalin in the gut mucosae by UDP-glucuronyl transferase and approximately half of the conjugate is excreted back into the gut. The absorbed baicalein are then extensively metabolized in the liver and excreted in urine and the feces via bile (Abe et al., 1990; Akao et al., 2000; Akao et al., 2004; Xing et al., 2005a; Lu et al., 2007). However, studies on the metabolism of wogonoside have not been performed.

Therefore, to understand the relationship between the metabolism of wogonoside from the rhizome of $\mathrm{SB}$, and its anti-pruritic effect, we anaerobically incubated it with human fecal microflora, identified its metabolite identified, and investigated its anti-pruritic effect in compound 48/80 or histamine-induced pruritic mice. 


\section{MATERIALS AND METHODS}

\section{Materials}

Azelastine, compound $48 / 80$, and histamine were purchased from Sigma Chem. Co. (St. Louis, MO, U.S.A.).

\section{Isolation of wogonoside from the rhizome of SB}

The cut and dried rhizomes of SB were purchased at KyungDong Market, Seoul, Korea (produced in Korea), and identified by one of the authors, NI Baek. The voucher specimen was deposited at the Grandual School of Biotechnology, Kyung Hee University. The cut and dried rhizomes $(1 \mathrm{~kg})$ were extracted with $80 \% \mathrm{MeOH}(4 \mathrm{~L} \times 2)$ at room temperature for $24 \mathrm{~h}$. The extracted solution was filtered through filter paper (No. 2) and concentrated by vacuum rotary evaporator. The concentrates were poured into $\mathrm{H}_{2} \mathrm{O}(3 \mathrm{~L})$ and extracted using EtOAc $(1 \mathrm{~L} \times 2)$ and $n-\mathrm{BuOH}$ $(1 \mathrm{~L} \times 2)$, successively. Each layer was evaporated to afford an EtOAc fraction (18 g), a $n-\mathrm{BuOH}$ fraction $(16 \mathrm{~g})$, and an aqueous fraction (SBW, $285 \mathrm{~g}$ ). A part of fraction SBW (140 g) was dissolved in $\mathrm{H}_{2} \mathrm{O}(2 \mathrm{~L})$ and acidified to $\mathrm{pH} 1$ by adding $2 \mathrm{~N} \mathrm{HCl}$, and stored at $10^{\circ} \mathrm{C}$ for one day. The fraction SBW $(140 \mathrm{~g})$ was dissolved with $50 \% \mathrm{MeOH}(430 \mathrm{ml})$ and stored at $10^{\circ} \mathrm{C}$ for one day. The precipitate was filtered through a glass filter and washed with cold $\mathrm{H}_{2} \mathrm{O}$ to yield purified wogonoside $(1.5 \mathrm{~g})$. These compounds were identified by comparison of their NMR data with those of the reported in literature (Takido et al., 1975; Tomimori et al., 1982; Lee et al., 2003).

Wogonoside (2) Yellow powder $\left(\mathrm{H}_{2} \mathrm{O}\right) ; \mathrm{mp} 194-196^{\circ} \mathrm{C}$;

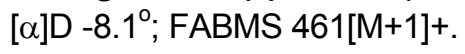

\section{Metabolism of wogonoside by human fecal microflora}

The human fecal specimens (about $30 \mathrm{~g}$ ) prepared according to a previous method (Lee et al., 2003) were collected in plastic cups $9 \mathrm{~h}$ after fasting, and then carefully mixed with a spatula and suspended with cold $270 \mathrm{ml}$ saline. The fecal suspension was centrifuged at $100 \times \mathrm{g}$ for $5 \mathrm{~min}$. The supernatant was then centrifuged at $10,000 \times \mathrm{g}$ for $20 \mathrm{~min}$. The resulting precipitates (about $3 \mathrm{~g}$ ) were used as a metabolic enzyme source for the assay of enzyme activity. The preparation and assay of the enzyme source were performed within $24 \mathrm{~h}$ at $4^{\circ} \mathrm{C}$.

To identify the metabolites of wogonoside by human intestinal bacteria, the reaction mixture contained $100 \mathrm{mg} / \mathrm{ml}$ of wogonoside and $2.5 \mathrm{~g}$ fresh human feces in a final volume of $500 \mathrm{ml}$ of anaerobic dilution medium. The mixture was incubated at $37^{\circ} \mathrm{C}$ for $20 \mathrm{~h}$. An aliquot ( $1 \mathrm{ml}$ ) of the reaction mixture was periodically extracted with ethyl acetate, concentrated, dissolved in $\mathrm{MeOH}$, and their metabo- lites were analyzed by LC-MS/MS (Agilent 1,200 series LC-MS/MS system consisting of a quaternary pump, a vacuum degasser, an autosampler, a thermostat column compartment, a diode array detector, and an Agilent G6410 triple quadrupole mass spectrometer with an electro spray ionization (ESI) source): column, ZORBAX Extend C18 $(100 \times 2.1 \mathrm{~mm}$ i.d., $1.8 \mu \mathrm{m}$, Agilent); elution solvent, a linear-gradient applied by $5 \%$ in solvent A to $95 \%$ B for $13 \mathrm{~min}$ (solvent $A-0.1 \%$ aqueous formic acid and solvent $B$ - acetonitrile); and elution rate, $0.3 \mathrm{ml} / \mathrm{min}$. Mass spectra was acquired in ESI mode using nitrogen gas at a temperature of $350^{\circ} \mathrm{C}$, flow rate of $10 \mathrm{~L} / \mathrm{min}$, nebulizer pressure of $45 \mathrm{psi}$, quadrupole temperature of $30^{\circ} \mathrm{C}$, and capillary voltage of $4,000 \mathrm{~V}$. The mass spectrometer was operated in positive mode with multiple reaction monitoring (MRM). The instrument was controlled and data were processed by Agilent MassHunter workstation software (Rev. B.01.00).

Each odd reaction mixture was evaporated to afford an EtOAc fraction $(38 \mathrm{mg})$. The ethyl acetate fraction was subjected to a MPLC equipped with a slica gel $100 \mathrm{C} 18$ column $(3 \times 50 \mathrm{~cm})$ - reversed phase eluted with $30 \%$ $\mathrm{MeOH}(5 \mathrm{~L})$. The EtOAc fraction of wogonoside gave the metabolite M1 (12 mg, wogonin). The metabolite was verified by comparison of its NMR data with those of the reported in literatures (Takido et al., 1975; Tomimori et al., 1982).

M1 (Wogonin) Pale yellow needles. mp 198-199oC, El-Mass $(\mathrm{m} / \mathrm{z}): 284$ [M ${ }^{+}$]. ${ }^{1} \mathrm{H}-\mathrm{NMR}\left(400 \mathrm{MHz}, \mathrm{C}_{5} \mathrm{D}_{5} \mathrm{~N}, \delta\right)$ : $12.35(1 \mathrm{H}, \mathrm{s}), 8.73(1 \mathrm{H}, \mathrm{s}), 8.07(2 \mathrm{H}, \mathrm{d}, \mathrm{J}=6.8 \mathrm{~Hz}), 7.61$ $(3 \mathrm{H}, \mathrm{m}), 7.04(1 \mathrm{H}, \mathrm{s}), 6.94(1 \mathrm{H}, \mathrm{s}), 3.88(3 \mathrm{H}, \mathrm{s}) .{ }^{13} \mathrm{C}-\mathrm{NMR}$ $\left(100 \mathrm{MHz}, \mathrm{C}_{5} \mathrm{D}_{5} \mathrm{~N}, \delta\right): 182.43(\mathrm{C}-4), 163.61$ (C-2), 151.38 (C-7), 156.08 (C-5), 149.25 (C-9), 132.42 (C-4'), 130.84 (C-1'), 129.43 (C-8, 3', 5'), 126.54 (C-2', 6'), 105.40 (C-3, 10), 98.98 (C-6), $61.37\left(\mathrm{OCH}_{3}\right)$.

\section{Animals}

The male ICR and BALB/c mice (18-22 g) were supplied from Charles River Orient Experimental Animal Breeding Center (Seoul, Korea). All animals were housed in wire cages at $20-22^{\circ} \mathrm{C}$, a relative humidity of $50 \pm 10 \%$ humidity, a frequency of air ventilation of $15-20$ times/h, and $12 \mathrm{~h}$ illumination (0.7:00-19:00; intensity, 150-300 Lux), fed standard laboratory chow (Charles River Orient Experimental Animal Breeding Center, Seoul Korea) and allowed water ad libitum. All procedures relating to the animals and their care conformed to the international guidelines 'Principles of Laboratory Animals Care' (NIH publication no. 85-23, revised 1985). 


\section{Pruritic behavioral experiments}

Before the experiment, the BALB/c (for compound 48/80 as a pruritic inducer) and ICR (for histamine) mice were put into acrylic cages $(22 \times 22 \times 24 \mathrm{~cm})$ for about $10 \mathrm{~min}$ for acclimation. The behavioral experiments were performed according to the method of Sugimoto et al. (1998). The rostral part of the skin on the back of mice was clipped, and $50 \mu \mathrm{g} / 50 \mu \mathrm{l}$ of compound $48 / 80$ or $300 \mu \mathrm{g} / 50 \mu \mathrm{l}$ of histamine for each mouse was intradermally injected with the use of a 29 gauge needle. The pruritic agents were dissolved in saline prior to injection. Control mice received a saline injection in the place of the pruritic agent. Immediately after the intradermal injection, the mice (one animal/cage) were put back into the same cage and, for the observation of pruritus; their behaviors recorded using an 8-mm video camera (SV-K80, Samsung, Seoul, Korea) under unmanned conditions. The pruritus of the injected site by the hind paws was counted and compared with that of other sites, such as the ears. Each mouse was used for only one experiment. The mice generally showed a pruritic frequency of several scratches per second, and a series of these behaviors were counted as one incident of the pruritus for $60 \mathrm{~min}$. Test agents were orally or intraperitoneally administered either $1 \mathrm{~h}$ or $5 \mathrm{~h}$ before treatment with the pruritic agent, histamine, in mice

The inhibition (\%) against pruritic reaction was calculated as follows: $100 \times$ [(the pruritic behavioral frequency of control group-that of normal group)-(that of test agent-treated group-normal group)]/(the pruritic frequency of control group - that of normal group).

\section{Statistics}

All the data were expressed as the mean \pm standard deviation, and statistical significance was analyzed by one way ANOVA followed by Student-Newman-Keuls test.

\section{RESULTS}

\section{Metabolism of wogonoside by human intestinal microflora}

To investigate the metabolite of wogonoside produced by human intestinal microflora, wogonoside was anaerobically incubated with human fecal microflora for $20 \mathrm{~h}$ and analyzed by LC-MS/MS. In the reaction mixture of wogonoside, one metabolite was observed. The metabolite possessed an MS peak at $m / z=285[\mathrm{M}+1]^{+}$. The metabolite of wogonoside was wogonin.

When the metabolic activity of wogonoside in five human fecal specimens was preliminarily assayed, its hydrolysis to its aglycone occurred in all specimens. The average of the hydrolyzing activities of wogonoside to wogonin was $6.9 \pm 5.1 \mathrm{nmol} / \mathrm{h} / \mathrm{mg}$ wet weight of fecal bacteria (Fig. 1).

\section{Effect of SB extract, wogonoside and wogonin on pruritic behaviors induced by compound $\mathbf{4 8 / 8 0}$ or histamine in mice}

SB extract inhibited compound 48/80-induced pruritic behavior in our preliminarly experiments. Therefore, the antipruritic effect of wogonoside, another main constituent of SB except baicalin, and its metabolite, wogonin, was in-

A

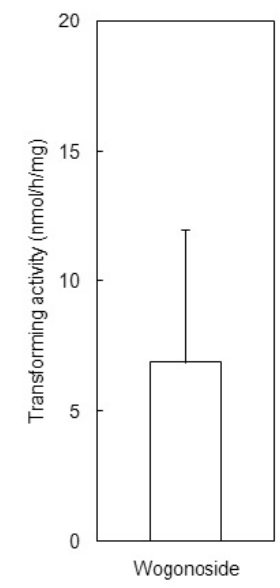

B
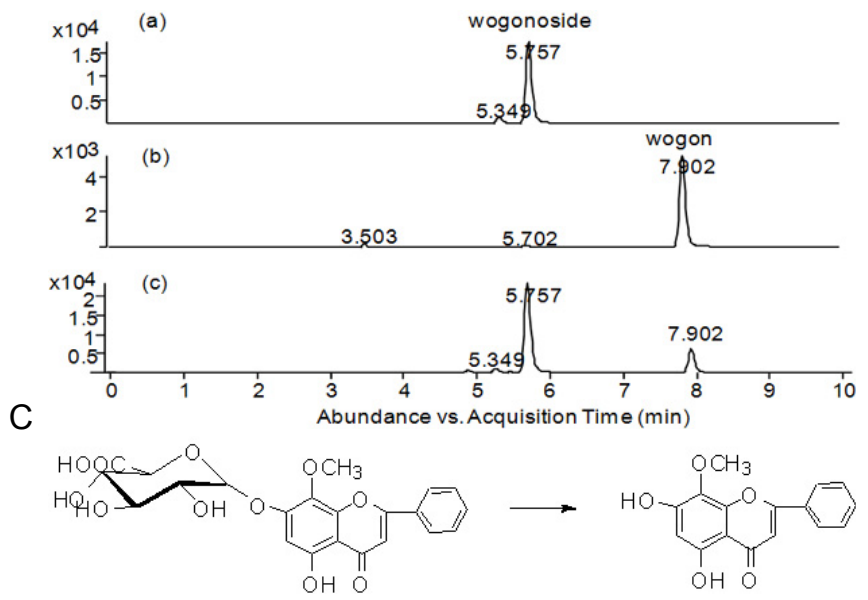

Fig. 1. Metabolic activity of wogonoside by human fecal microflora (A), its metabolic profile by LC- MS/MS analysis (B) and its proposed pathway (B). LC-MS/MS chromatogram of the reaction mixture incubated wogonoisde with human fecal suspension: (a) wogonoside standard (7.75 $\mathrm{min})$, (b) wogonin standard (7.90 $\mathrm{min})$, (c) $5 \mathrm{~h}$ after incubation after incubation of wogonoside with human fecal suspension. LC- MS/MS system (Agilent 1200 series): column, ZORBAX Extend C18 $(100 \times 2.1 \mathrm{~mm}$ i.d., $1.8 \mu \mathrm{m}$, Agilent); elution solvent, a linear- gradient applied by $5 \%$ in solvent $A$ to $95 \%$ B for 13 min (solvent $A-0.1 \%$ aqueous formic acid and solvent $B$ - acetonitrile); and elution rate, $0.3 \mathrm{ml} / \mathrm{min}$. $\rightarrow$, main pathway; $\rightarrow$, minor pathway. 

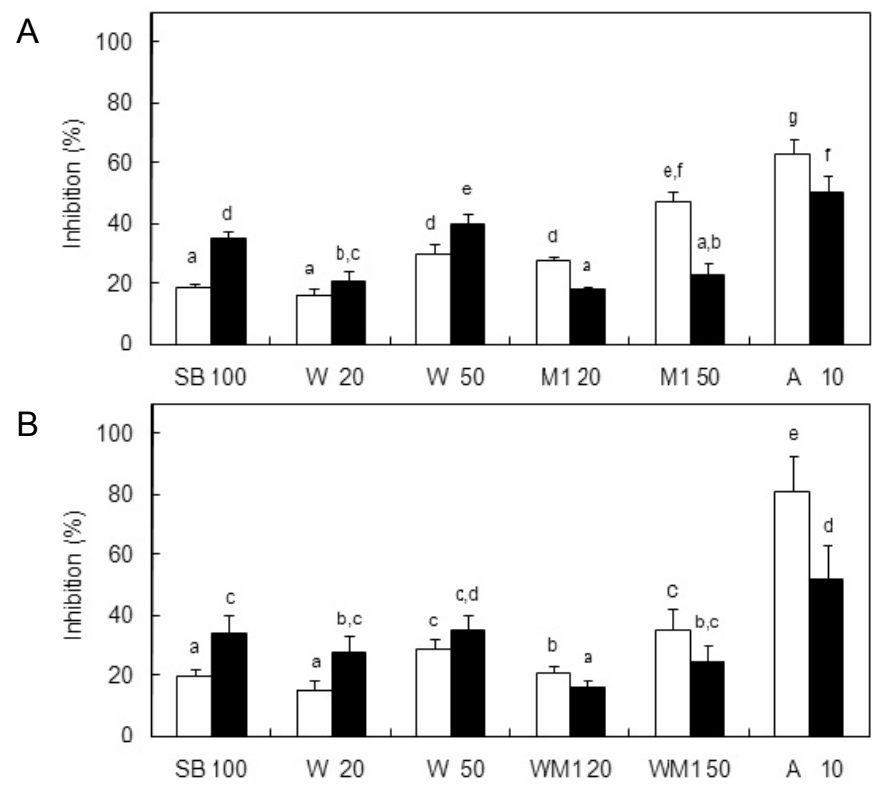

Fig. 2. Inhibitory effects of orally administered Scutellariae radix extract, wogonoside, and its metabolite, and azelastine, on compound 48/80 (A)- and histamine (B)-induced pruritus in mice. The pruritus was induced by compound $48 / 80$ in BALB/c mice or histamine in ICR mice. Mice were treated with or without oral administration of test agents $(10,20,50$ and/or $100 \mathrm{mg} / \mathrm{kg}) 1 \mathrm{~h}$ (white bar) or $5 \mathrm{~h}$ (black bar) before the intradermal injection of 50 $\mu \mathrm{g} / 50 \mu \mathrm{l}$ of compound $48 / 80$ or $300 \mu \mathrm{g} / 50 \mu \mathrm{l}$ of histamine into the shaved back skin of mice: SB100, the radix extract of Scutellaria baicalensis (SB, $100 \mathrm{~m} / \mathrm{kg}$ ); W 20, $20 \mathrm{mg} / \mathrm{kg}$ wogonoside; W 50, $50 \mathrm{mg} / \mathrm{kg}$ wogonoside; M1 20, $20 \mathrm{mg} / \mathrm{kg}$ wogonin; M1 50, 50 $\mathrm{mg} / \mathrm{kg}$ wogonin; A 10, $10 \mathrm{mg} / \mathrm{kg}$ azelasitine. Numbers of pruritic behavior frequency of normal group (treated with saline alone) and control groups (treated with compound $48 / 80$ or histamine) for $1 \mathrm{~h}$ were $3 \pm 1,222 \pm 21$, and $89 \pm 5$, respectively. The values indicate mean \pm S.D. $(n=6)$. ${ }^{\text {a, }, ~}, c, d, e, f, g$ Items with the same letter are not significantly different $(p>0.05)$.

vestigated on compound 48/80-induced experimental pruritic mice (Fig. 2A). When compound 48/80 was injected into mice, prurirtic behavior was significantly induced. When wogonoside was orally administered $1 \mathrm{~h}$ or $5 \mathrm{~h}$ before the treatment with a pruritic agent compound $48 / 80$, it inhibited the pruritic behavior. Wogonoside orally administered $5 \mathrm{~h}$ before the treatment with compound $48 / 80$ more potently inhibited the pruritus than one administered $1 \mathrm{~h}$ before. Wogonoside at a dose of $50 \mathrm{mg} / \mathrm{kg}$ showed the anti-pruritic effect in compound 48/80-treated mice, with inhibitions of $31 \%$ and $40 \%$, respectively. However, wogonin orally administered $1 \mathrm{~h}$ before the treatment with compound 48/80 more potently showed the anti-pruritic effect than one treated $5 \mathrm{~h}$ before. Wogonin orally administered $1 \mathrm{~h}$ after pruritic agent, inhibited the pruritic behaviors by $47 \%$.

Orally administered wogonoside and wogonin inhibited

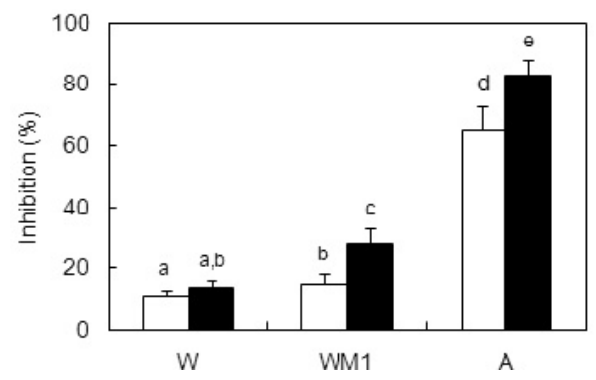

Fig. 3. Inhibitory effects of intraperitoneally administered wogonoside, its metabolite, and azelastine, on histamine-induced pruritus in mice. The pururitus was induced by histamine in ICR mice. Mice were treated with or without intraperitoneal administration of test agents [5 (white bar) and $10 \mathrm{mg} / \mathrm{kg}$ (black bar)] $1 \mathrm{~h}$ before the intradermal injection of $300 \mu \mathrm{g} / 50 \mu \mathrm{l}$ of histamine into the shaved back skin of mice. W: wogonoside, M1: wogonin, A: azelasitine. Numbers of pruritic behavior frequency of normal group (treated with histamine) and control group (treated with saline alone) for $1 \mathrm{~h}$ were $85 \pm 4$, and $2 \pm 1$, respectively. The values indicate mean \pm S.D. $(n=6) .{ }^{a, b, c, d, e}$ Items with the same letter are not significantly different $(p>0.05)$.

histamine-induced pruritus (Fig. 2B). Wogonoside orally administered $5 \mathrm{~h}$ before the treatment with histamine, more potently inhibited the scratching behaviors than one treated $1 \mathrm{~h}$ before. Wogonoside at a dose of $50 \mathrm{mg} / \mathrm{kg}$ inhibited the pruritus by $29 \%$ and $35 \%$, respectively. Its metabolite, wogonin orally administered $1 \mathrm{~h}$ before the treatment with histamine, more potently inhibited the pruritus than one administered $5 \mathrm{~h}$ before.

The inhibitory effect of intraperitoneally administered wogonoside and its metabolite, wogonin, in histamine-induced pruritic mice was measured (Fig. 3). Although all these agents, wogonoside and wogonin, inhibited the pruriritic behavior, wogonin was more effective than baicalin.

\section{DISCUSSION}

Pruritus, an unpleasant cutaneous sensation which provokes the desire to scratch, can be local or widespread and is associated with atopic dermatitis, uriticaria, cholestasis, uraemia, etc. Many endogenous amines, proteases, growth factors, neuropeptides, opioids, ecosanoids and cytokines can act as pruritogens (Lerner, 1944; Hagemark, 1995; Schmeiz et al., 1997). Pruritus can cause skin lesions and contribute to severe psychological disturbances (Raiford, 1995). Therefore, inhibition of this response is beneficial for improving the quality of life. However, there is no specific remedy available for this common symptom. As part of a screening program aimed at discovering anti-pruritic agents from natural products, we found that SB potently inhibit compound 48/80-induced pruritus. Many re- 
searchers also reported the inhibitory effects of SB and baicalin against allergic diseases, asthma and inflammation (Koda et al., 1970; Liaw et al., 1999; Taniguchi et al., 2000). However, anti-allergic or anti-pruritic effect of wogonoside, a main constituent of SB, was not studied, whereas its anti-inflammatory effects were reported. In the present study, we found its metabolite, wogonin, by human fecal microflora. Wogonoside was metabolized to wogonin, which was not transformed any further during a $24 \mathrm{~h}$ incubation period. The metabolite may be absorbed from the intestine into the blood. This suggestion is supported by the reports of Xing et al. (2005a, 2005b) that baicalein and wogonin conjugates were detected in the blood of rats orally treated with baicalin and wogonoside, respectively.

Next we evaluated the anti-pruritic effects of wogonoside and its metabolite in compound $48 / 80$ or histamine-induced pruritic mice. Compound $48 / 80$ or histamine were intradermally injected into the rostral part of the back skin of BALB/C and ICR mice, and the pruritic behaviors evaluated for $60 \mathrm{~min}$. The scratching frequencies for the $60 \mathrm{~min}$ period for each inducer increased in a dose?dependent manner. These scratching agents more potently induced pruritic behaviors in ICR mice than in BALB/c mice, which are in agreement with a previous report (Inagaki et al., 2001). Particularly, compound $48 / 80$ caused vigorous scratching behavior in ICR mice. The accurate counting of scratching behavior frequencies proved too difficult. However, BALB/c mice were dull for histamine. Therefore, in the present study, BALB/c mice were used in our compound 48/80-induced pruritic mouse model and ICR were use in our histamine-induced pruritic mouse model. SB and its constituent, wogonoside, showed anti-pruritic effects in compound $48 / 80$ or histamine-stimulated mice. Orally administered wogonoside at $5 \mathrm{~h}$ before the treatment with scratching agents showed the potent anti-pruritic effects than one treated at $1 \mathrm{~h}$ before. However, wogonin showed more potent inhibition, when treated at $1 \mathrm{~h}$ before the treatment with scratching agents than when treated at 5 before. These results suggest that, to express the anti-pruritic effect of wognoside, it should be metabolized by intestinal microflora, but that of wogonin may be independent on its metabolism by intestinal microflora. This suggestion support that the anti-pruritic effect of orally administered wogonoside was more potent than that of intraperitoneally administered one, whereas intraperitoneally administered wogonin showed more potent anti-pruritic effect than orally treated one. These results suggest that orally administered wogonoside may be metabolized to wogonin by intestinal microflora. Following absorption by intestinal microflora, its metabolite may express pharmaco- logical effects, such as anti-pruritic, anti-inflammatory and antioxidant effects.

Finally, these findings suggest that SB and its constituent, baicalin, can attenuate the pruritus and, and that its anti-pruritic effects may be dependent on its metabolism by intestinal microflora.

\section{ACKNOWLEDGMENTS}

The authors are grateful to Dr Sang-Jun Han for assisting with the metabolite analysis by LC-MS/MS.

\section{REFERENCES}

Abe, K., Inoue, O. and Yumioka, E. (1990). Biliary excretion of metabolites of baicalin and baicalein in rats. Chem. Pharm. Bull. 38, 209-211.

Akao, T., Kawabata, K. and Yanagisawa, E. (2000). Baicalin, the predominant flavone glucuronide of scutellariae radix, is absorbed from the rat gastrointestinal tract as the aglycone and restored to its original form. J. Pharm. Pharmacol. 52, 1563-1568.

Akao, T., Dakashita, Y., Hanada, M., Goto, H., Shimada, Y. and Terasawa, K. (2004). Enteric excretion of baicalein, a flavone of Scutellariae Radix, via glucuronidation in rat: involvement of multidrug resistance-associated protein 2. Pharm. Res. 21, 2120-2126.

Akao, T., Kida, H., Kanaoka, M., Hattori, M. and Kobashi, K. (1998). Intestinal bacterial hydrolysis is required for the appearance of compound $\mathrm{K}$ in rat plasma after oral administration of ginsenoside Rb1 from Panax ginseng. $J$. Pharm. Pharmacol. 50, 1155-1160.

Chou, T. C., Chang, L. P., Li, C. Y., Wong, C. S. and Yang, S. P. (2003). The antiinflammatory and analgesic effects of baicalin in carrageenan-evoked thermal hyperalgesia. Anesth. Analq. 17, 1724-1729.

Hagermark, O. (1955). Itch mediators. Semin. Dermatol. 14, 271-276.

Inagaki, N., Nagao, M., Kawasaki, H., Kim, J. F. and Nagai, H. (2001). Scratching behavior in various strains of mice. Skin Pharmacol. Appl. Skin Physiol. 14, 87-96.

Jang, S. I., Kim, H. J., Hwang, K. M., Jekal, S. J., Pae, H. O., Choi, B. M., Yun, Y. G., Kwon, T. O., Chung, H. T. and Kim, Y. C. (2003). Hepatoprotective effect of baicalin, a major flavone from Scutellaria radix, on acetaminophen-induced liver injury in mice. Immunopharmacol. Immunotoxicol. 25, 585-594.

Kim, D. H., Cho, K. H., Moon, S. K., Kim, Y. S., Kim, D. H., Choi, J. S. and Chung, H. Y. (2005). Cytoprotective mechanism of baicalin against endothelial cell damage by peroxynitrite. $J$. Pharm. Pharmacol. 57, 1581-1590.

Kobashi, K. and Akao, T. (1997). Relation of intestinal bacteria to pharmacological effects of glycosides. Bioscience Microflora 16, 1-7.

Koda, A., Nagai, H. and Wada, H. (1970). Pharmacological actions of baicalin and baicalein. 2. On passive anaphylaxis. Nippon Yakurigaku Zasshi 66, 237-247. 
Kuraishi, Y., Nagasawa, T., Hayashi, K. and Satoh, M. (1995) Scratching behavior induced by pruritogenic but not algesiogenic agents in mice. Eur. J. Pharmacol. 275, 229-233.

Lee, D. K., Kim, Y. S., Ko, C. N., Cho, K. H., Bae, H. S., Lee, K. S., JKim. J., Park, E. K. and Kim, D. H. (2003). Fecal metabolic activities of herbal components to bioactive compounds. Arch. Pharm. Res. 25, 165-169.

Lerner, E. A. (1994) "Itch: mechanisms and management of pruritis," ed. By Berhard J., McGraw-Hill, New York, pp 23-25.

Liaw, J., Gau, Y. Y. and Chao, Y. C. (1999). Effect of baicalin on tracheal permeability in ovalbumin (OA)-sensitized guinea pigs. Pharm. Res. 16, 1653-1657.

Lim, B. O. (2003). Effects of wogonin, wogonoside, and 3,5, $7,2^{\prime}, 6$ '-pentahydroxyflavone on chemical mediator production in peritoneal exduate cells and immunoglobulin $\mathrm{E}$ of rat mesenteric lymph node lymphocytes. J. Ethnopharmacol. 84, 23-29.

Lu, T., Song, J., Huang, F., Deng, Y., Xie, L., Wang, G. and Liu, $X$. (2007). Comparative pharmacokinetics of baicalin after oral administration of pure baicalin, Radix scutellariae extract and Huang-Lian-Jie-Du-Tang to rats. J. Ethnopharmacol. 110, 412-418.

Raiford, D. S. (1995). Pruritus of chronic cholestasis. QJM 88, 603-607.

Schmeiz, M., Schmidt, R., Bickel, A., Handerker, H. O. and Torebjork, H. E. (1997). Specific C-receptors for itch in human skin. J. Neurosci. 17, 8003-8008.

Sugimoto, Y., Umakoshi, K., Nojiri, N. and Kamei, C. (1998). Effects of histamine $\mathrm{H} 1$ receptor antagonists on compound 48/80-induced scratching behavior in mice. Eur. J.
Pharmacol. 351, 1-5.

Taniguchi, C., Homma, M., Takano, O., Hirano, T., Oka, K., Aoyagi, Y., Niitsuma, T. and Hayashi, T. (2000). Pharmacological effects of urinary products obtained after treatment with saiboku-to, a herbal medicine for bronchial asthma, on type IV allergic reaction. Planta Med. 66, 607-611.

Takido, M., Aimi, M., Takahashi, S., Yamamouchi, S., Torii, H. and Dohi, S. (1975). Studies on the constituents in the water extracts of crude drugs. I. On the roots of Scutellaria baicalensis Georgi (Wōgon) (1). Yakhagaku Zasshi 95, 108-113.

Tomimori, T., Miyaichi, Y. and Kizu, H. (1982). On the flavonoid constituents from the roots of Scutellaria baicalensis Georgi. I. Yakugaku Zasshi 102, 388-391.

Wu, S., Sun, A. and Liu, R. (2005). Separation and purification of baicalin and wogonoside from the Chinese medicinal plant Scutellaria baicalensis Georgi by high-speed counter-current chromatography. J. Chromatogr. A. 1066, 243-247.

Xing, J., Chen, X. Y. and Zhong, D. F. (2005a). Absorption and enterohepatic circulation of baicalin in rats. Life Sci. 78, 140-146.

Xing, J., Chen, X. Y., Sun, Y. M., Luan, Y. and Zhong, D. F. (2005b). Interaction of baicalin and baicalein with antibiotics in the gastrointestinal tract. J. Pharm. Pharmacol. 57, 743-750.

Yim, J. S., Kim, Y. S., Moon, S. K., Cho, K. H., Bae, H. S., Kim, J. J, Park, E. K. and Kim, D. H. (2004). Metabolic activities of ginsenoside Rb1, baicalin, glycyrrhizin and geniposide to their bioactive compounds by human intestinal microflora. Biol. Pharm. Bull. 27, 1580-1583.

Zhu, Y. P. (1998). Chinese Materia Medica. Harwood Academic Publichers, Australia. pp.127-135. 